Génét. Sél. Evol., 1985, 17 (3), 311-330

\title{
Distribution et association des inversions chromosomiques dans trois populations naturelles de Drosophila melanogaster de France, Tunisie et Congo
}

\author{
Sylvie AULARD *,**, Françoise LEMEUNIER * \\ * C.N.R.S., Laboratoire de Biologie et Génétique évolutives, \\ F 91190 Gif-sur-Yvette. \\ ** Université Pierre et Marie Curie, Paris.
}

\begin{abstract}
Résumé
Le polymorphisme chromosomique de 3 populations naturelles de Drosophila melanogaster originaires de régions tempérée (France), méditerranéenne (Tunisie) et tropicale (Congo) a été analysé. Les résultats obtenus sont les suivants : 1 . Les 17 inversions décelées, dont 8 cosmopolites, sont paracentriques et localisées sur les 2 grands autosomes. 2. La population de Tunisie est celle qui présente le plus de variabilité; celle du Congo montre un faible polymorphisme analogue à celui de la population française. 3. La distribution des inversions cosmopolites entre les individus de cette dernière population est aléatoire, mais il existe un excès de mouches ne possédant qu'un seul remaniement. A l'inverse, cette catégorie est significativement minoritaire dans l'échantillon du Congo. Une répartition non aléatoire est également mise en évidence dans la population tunisienne. 4. Des déséquilibres de liaison existent pour les inversions cosmopolites du chromosome 2 en Tunisie, pour celles du chromosome 3 au Congo. 5. Des associations non aléatoires entre les différentes séquences chromosomiques des 2 autosomes sont décelées dans les échantillons de France et du Congo.
\end{abstract}

Mots clés : Polymorphisme d'inversion chromosomique, Drosophila melanogaster, déséquilibre de liaison, association interchromosomique.

\section{Summary}

Nature, frequencies and associations of chromosome inversions in French, Tunisian and Congolese natural populations of Drosophila melanogaster

The chromosome inversion polymorphism of 3 natural populations of Drosophila melanogaster from temperate (France), mediterranean (Tunisia) and tropical (Congo) regions has been studied. All 17 of the inversion types found are paracentric and autosomal, but only 8 of these, one on each of the major autosome arms, are cosmopolitan. The Tunisian population shows the greatest variability. The Congolese population shows a slight polymorphism, similar to the French population. A random distribution of cosmopolitan inversions between individuals of the French population is observed, but there is an excess of flies carrying only one rearrangement. On the contrary, the frequency of such individuals is lower than expected in the Congolese population. A non-random distribution is also detected in the Tunisian lines. Linkage disequilibrium exists between pair of cosmopolitan inversions of the 
second chromosome in Tunisia, and of the third chromosome in Congo. Non-random associations of the second and third chromosomes bearing inversions are seen in the French and Congolese populations.

Key words : Inversion polymorphism, Drosophila melanogaster, linkage disequilibrium, associations between inversions.

\section{Introduction}

Les populations naturelles de nombreuses espèces de Diptères, notamment les Drosophiles, présentent un polymorphisme d'inversion chromosomique. Jusqu'à une date récente, le polymorphisme de Drosophila melanogaster semblait être réduit. Mais, en moins de 10 ans, le nombre des inversions décrites dans les populations naturelles s'est tellement accru que l'espèce apparaît actuellement comme l'une des plus variables parmi les Drosophiles, avec près de 300 remaniements connus. Ces inversions ont été classées en fonction de leur fréquence et de leur répartition géographique selon une nomenclature précise (Ashburner \& Lemeunier, 1976 ; Mettler et al., 1977 ; Lemeunier et al., 1985).

Plusieurs études semblent prouver que ces remaniements font l'objet d'une sélection naturelle : existence de clines latitudinaux (METTLER et al., 1977 ; KNIBB et al., 1981), de fluctuations saisonnières (INOUE, 1979; STALKER, 1980), de déséquilibres entre inversions, intra et interchromosomiques (AlaHIOTIS et al., 1976 ; STALKER, 1976 ; LANGLEY et al., 1977 ; INOUE \& WATANABE, 1979 ; KNIBB et al., 1981 ; YAMAZAKI et al., 1984), de corrélations avec la niche écologique (STALKER, 1976), avec des facteurs climatiques (STALKER, 1980 ; KNIBB, 1982).

Jusqu'à présent, la plupart des travaux ont porté sur des populations américaines, japonaises et australiennes. Il n'existe que très peu de résultats pour l'Europe, l'Afrique du Nord et aucun pour l'Afrique tropicale. Or cette région est sans doute le berceau de l'espèce (TsaCAS \& LACHAISE, 1974; TsaCAS, 1979 ) et il était important d'étudier des populations qui demeurent vraisemblablement dans un état proche de celui que présentait l'espèce avant sa propagation. Il était également intéressant de savoir si les variations latitudinales connues en Amérique, Australie et Asie se retrouvaient entre l'Europe et l'Afrique. A cet effet, trois populations, originaires de régions tempérée (France), méditerranéenne (Tunisie) et tropicale (Congo) ont été étudiées afin de déterminer leur variabilité chromosomique. Les caractéristiques analysées concernent la distribution des inversions cosmopolites par individu, leur déséquilibre de liaison et les associations entre remaniements cosmopolites non liés.

\section{Matériel et méthodes}

Les trois échantillons analysés sont constitués chacun de 50 lignées isofemelles :

(1) Villeurbanne (France), Automne 1980 (25 lignées) et Eté 1981 (25 lignées),

(2) Brazzaville (Congo), Printemps 1981, (3) Nasr'Allah (Tunisie), Automne 1981. 


\section{A. Estimation de la fréquence des inversions}

Un mâle de chaque lignée est croisé avec une femelle vierge d'une souche de référence $D$. melanogaster Canton $\mathrm{S}$, homozygote pour la séquence standard. La présence de remaniements chromosomiques apportés par le gamète paternel est mise en évidence par une anomalie de l'appariement des homologues chez les larves de première génération. Six larves au minimum sont examinées afin de déterminer la composition génotypique du mâle étudié.

Les préparations des chromosomes polytènes se font après dissection des glandes salivaires des larves de troisième stade, puis fixation (éthanol absolu - acide propionique, 3 : 1) et coloration par l'orcéïne - carmin - propionique (ASHBURNER, 1967).

Les points de cassure des inversions sont identifiés sur photographies à l'aide des cartes de référence de Bridges (1935) et LeFEVRe (1976). La fréquence des inversions dans ces échantillons est calculée par bras chromosomique.

\section{B. Test d'égalité de 2 proportions et d'indépendance de 2 caractères}

Chaque bras chromosomique présente 2 variantes complémentaires : séquence inversée (In) et séquence standard (St). Leurs proportions et leurs associations intra et interchromosomiques ont été testées par le traitement exact de Fisher des tables $2 \times 2$ en raison de la faible fréquence des inversions dans la plupart des cas.

\begin{tabular}{c|c|c|c}
\hline & A 1 & A 2 & \\
\hline B 1 & x 11 & x 12 & x 10 \\
\hline B 2 & x 21 & x 22 & x 20 \\
\hline & x 01 & x 02 & N \\
\hline \hline
\end{tabular}

A 1 et $\mathbf{A} 2$ désignent soit les 2 variantes du caractère $A$ (InA et StA), soit 2 populations dont l'homogénéité est testée.

B 1 et B 2 sont les deux variantes du caractère B (InB et StB).

$\times 11, \times 12, \times 21$ et $\times 22$ sont les fréquences observées.

$\mathrm{x} 01=\mathrm{x} 11+\mathrm{x} 21$

$\mathrm{x} 02=\mathrm{x} 12+\mathrm{x} 22$

$\mathrm{x} 10=\mathrm{x} 11+\mathrm{x} 12$

x $20=\times 21+\times 22$

$\mathrm{N}$ est le nombre total d'observations indépendantes :

$\mathrm{N}=\times 10+\times 20=\times 01+\times 02$. 
La probabilité exacte d'observer une telle répartition, avec des fréquences marginales fixées, est donnée par la distribution hypergéométrique :

$$
P(X=x \quad 11)=\frac{\left(\begin{array}{lll}
x & 11 \\
x & 01
\end{array} \times\left(\begin{array}{lll}
x & 12 \\
x & 02
\end{array}\right.\right.}{\left(\begin{array}{lll}
x & 10 \\
N
\end{array}\right.}
$$

Pour un test statistique de l'hypothèse nulle, les écarts plus extrêmes que ceux observés doivent être pris en considération et l'hypothèse de l'indépendance des 2 caractères $A$ et $B$, ou d'homogénéité des populations $A 1$ et $A 2$ pour le caractère $B$, est rejetée au niveau de probabilité $\alpha$ si :

$$
\sum_{y 11} P(X=x 11)=\sum_{y} \frac{x 01 ! x 02 ! x 10 ! x 20 !}{y 11 ! y 12 ! y 21 ! y 22 ! N !} \leqslant \alpha / 2
$$

la sommation étant faite :

pour les valeur y $11 \leqslant$ x 11 si : x $11 \leqslant \mathrm{E}(\mathrm{x} 11)$

c'est-à-dire si : x $11 . \times 22-\times 12 . \times 21 \leqslant 0$

pour les valeurs y $11 \supseteq \mathrm{x} 11$ si : x $11 \supseteq \mathrm{E}(\mathrm{x} 11)$

c'est-à-dire si : x 11 × $22-$ x 12 × $21 \supseteq 0$

(Références : Siegel, 1956 ; EveritT, 1977).

C. Coefficient de déséquilibre et coefficient de corrélation de point

Entre 2 inversions (InA et InB) portées par les bras gauche et droit d'un même chromosome ou de 2 chromosomes différents et les séquences standard respectives (StA et StB), 4 types de combinaison peuvent se présenter : InA . InB, InA . StB, StA . InB et StA . StB, de fréquence relative $\mathrm{f} 1$, f 2 , f 3 et $\mathrm{f} 4$ respectivement :

$$
\begin{aligned}
& \text { f } 1=\times 11 / N \\
& \text { f } 2=\times 21 / N \\
& \text { f } 3=\times 12 / N \\
& \text { f } 4=\times 22 / N
\end{aligned}
$$

Le degré de liaison entre les inversions est estimé par le coefficient de déséquilibre $\mathbf{D}$ (covariance entre les inversions) et le coefficient de corrélation de point $\mathbf{R}$.

$$
\begin{gathered}
D=f 1 . \mathrm{f} 4-\mathrm{f} 2 . \mathrm{f} 3 \\
R=\frac{\mathrm{D}}{\sqrt{\mathrm{p}(1-\mathrm{p}) \bar{q}(1-\mathrm{q})}}
\end{gathered}
$$

$p$ et $q$ étant les fréquences relatives des inversions $A$ et $B,(1-p)$ et $(1-q)$ celles des séquences standard, 
d'où :

$$
\mathrm{R}=\frac{\mathrm{x} 11 . \times 22-\times 12 . \times 21}{\sqrt{\mathrm{x} 01 . \times 02 . \times 10 . \times 20}}
$$

$R$ est positif lorsqu'il existe un excès d'associations entre les inversions et négatif dans le cas contraire.

\section{Résultats}

\section{A. Nature et fréquence des remaniements}

Le caryotype de $D$. melanogaster comporte une paire de chromosomes sexuels $\left(\mathrm{n}^{\circ} 1\right)$, 2 paires de grands autosomes métacentriques $\left(n^{\circ} 2\right.$ et 3$)$ et une paire de chromosomes punctiformes $\left(n^{\circ} 4\right)$.

Les remaniements chromosomiques se localisent essentiellement sur les bras des grands autosomes. Leur répartition géographique a permis à AsHBURNER et LEMEUNIER (1976) et à MetTLer et al. (1977) de distinguer 4 classes d'inversion :

(1) Les inversions cosmopolites communes :

$\operatorname{In}(2 \mathrm{~L}) \mathrm{t}$ et $\operatorname{In}(2 \mathrm{R}) \mathrm{NS}$ sur les bras gauche et droit du chromosome 2 . $\operatorname{In}(3 \mathrm{~L}) \mathrm{P}$ et In(3R)P sur les bras gauche et droit du chromosome 3.

Elles sont présentes pratiquement dans toutes les populations naturelles, avec des fréquences en général supérieures à 5 p. 100.

(2) Les inversions cosmopolites rares :

Elles sont également largement répandues mais leur distribution est plus restreinte et leur fréquence plus faible que celles des cosmopolites communes : In(2L)NS, $\operatorname{In}(2 \mathrm{R}) \mathrm{Cy}, \operatorname{In}(3 \mathrm{~L}) \mathrm{M}, \operatorname{In}(3 \mathrm{R}) \mathrm{C}, \operatorname{In}(3 \mathrm{R}) \mathrm{K}, \operatorname{In}(3 \mathrm{R}) \mathrm{Mo}$ et $\operatorname{In}(3 \mathrm{R}) \mathrm{M}$.

La localisation de ces remaniements sur les chromosomes polytènes est donnée dans la figure 1.

(3) Les inversions endémiques récurrentes :

Il n'existe que 3 inversions de ce type. Elles persistent dans une même population durant plusieurs années ou dans quelques localités adjacentes.

(4) Les inversions endémiques uniques :

C'est la très grande majorité des remaniements naturels. Découverts chez un seul individu, ils ne sont généralement plus retrouvés ultérieurement dans la même population.

Dans les 3 échantillons analysés, 17 inversions différentes, dont 8 cosmopolites, ont été détectées, 7 sur le chromosome 2 et 10 sur le chromosome 3 . Il s'agit d'inversions paracentriques, affectant tous les bras des grands autosomes. A l'exception des remaniements cosmopolites dont la localisation est bien connue et admise, elles sont désignées par leurs points de cassure dans la liste suivante : 


\section{CHROMOSOME 2}

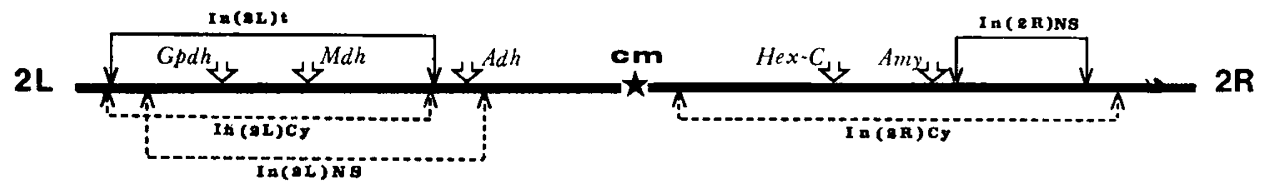

CHROMOSOME 3

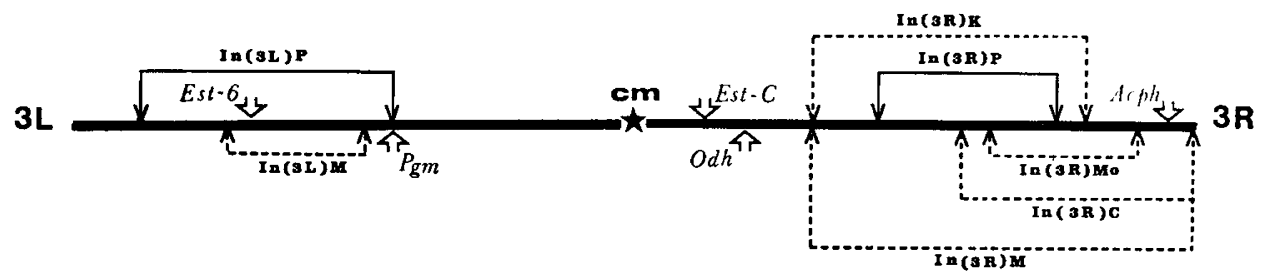

FIr. 1

Localisation cytogénétique des inversions cosmopolites, de In (2L)Cy et de quelques locus enzymatiques sur les chromosomes polytènes de D. melanogaster (en traits pleins : inversion cosmopolite commune; en pointillés : inversion cosmopolite rare).

Cytogenetic locations in the polytene chromosomes of $\mathbf{D}$. melanogaster of the cosmopolitan inversions, In $(2 L) C y$ and allozyme loci

(solid lines : common cosmopolitan inversion; dotted lines : rare cosmopolitan inversion).

Chromosome 2, bras gauche : $(2 \mathrm{~L})$

1. In(2L)t, cosmopolite commune (Vill., Brazz., Nasr'.,).

2. In(2L)Cy22L1 . 2 ; 33F5-34A1, endémique (Nasr'.). (Voir commentaires dans le texte.

3. In(2L)30A1 . $2 ; 33$ A3-8, nouvelle endémique, détectée dans deux lignées de Nasr'Allah.

Chromosome 2, bras droit : (2R)

4. In(2R)NS, cosmopolite commune (Vill., Brazz., Nasr'.).

5. In(2R)Cy, cosmopolite rare (Nasr'.).

6. In(2R) $44 \mathrm{D} ; 45 \mathrm{E}$, nouvelle endémique (Vill.).

7. $\operatorname{In}(2 \mathrm{R}) 45 \mathrm{~F} 3-8 ; 59 \mathrm{~B}$, nouvelle endémique (Nasr'.).

Chromosome 3, bras gauche : (3L)

8. In(3L)P, cosmopolite commune (Vill., Brazz.).

9. In(3L)65B ; 78D, nouvelle endémique (Vill.).

10. In $(3 \mathrm{~L}) 67 \mathrm{C} ; 70 \mathrm{~B}$, nouvelle endémique détectée deux fois, dont une incluse dans une inversion $\operatorname{In}(3 \mathrm{~L}) \mathrm{P}$ (Vill.).

11. In(3L)69A-B ; 73A, nouvelle endémique (Nasr'.).

12. In(3L)69A; 79E5-8, nouvelle endémique (Nasr'.). 


\section{Chromosome 3, bras droit : (3R)}

13. In(3R)P, cosmopolite commune (Vill., Brazz.).

14. In(3R)K, cosmopolite rare (Vill., Brazz.).

15. In(3R)C, cosmopolite rare (Vill., Brazz., Nasr'.).

16. In(3R)M, cosmopolite rare (Nasr'.).

17. In(3R)86E;99B, nouvelle endémique (Brazz.) détectée avec une inversion In(3R)P sur le même chromosome.

L'inversion In(2L)Cy n'était décrite jusqu'à présent que dans des souches de laboratoire. Cependant, à cause de leur grande similitude, il est possible que cette inversion ait été confondue parfois avec $\operatorname{In}(2 \mathrm{~L}) \mathrm{t}$, ce qui expliquerait son absence apparente d'autres populations naturelles. Mais en raison du doute qui subsiste, nous préférons la désigner comme "endémique » en dépit de sa fréquence relativement élevée dans l'échantillon de Tunisie qui pourrait la faire entrer dans la catégorie des inversions cosmopolites rares.

L'homogénéité des 2 échantillons de Villeurbanne quant aux proportions de chaque inversion cosmopolite et aux taux de remaniement des bras et des chromosomes a été testée par la loi hypergéométrique. Aucune différence significative n'a été trouvée, les probabilités étant toutes supérieures à $\alpha / 2=0,025$. Les données ont été regroupées. Les inversions endémiques ne donnent lieu à aucun polymorphisme durable. A quelques exceptions près, ce sont des événements uniques qui sont rapidement éliminés des populations naturelles. Pour cette raison, et en accord avec KNIBB et al. (1981), l'aspect quantitatif du polymorphisme chromosomique est étudié en ne tenant compte que des inversions cosmopolites. Les endémiques sont regroupées avec les séquences standard, à l'exception de $\operatorname{In}(2 \mathrm{~L}) \mathrm{Cy}$.

Les fréquences des inversions cosmopolites sont données dans le tableau 1. Pour plus de simplicité, ces dernières sont désignées par $2 \mathrm{Lt}$ pour $\operatorname{In}(2 \mathrm{~L}) \mathrm{t}, 2 \mathrm{RNS}$ pour In(2R)NS...

Le tableau 2 montre les probabilités obtenues pour le test d'homogénéité des échantillons. Villeurbanne et Brazzaville présentent les mêmes remaniements cosmopolites communs et rares : 2Lt, 2RNS, 3LP, 3RP, 3RC et 3RK. Les 2 premiers, sur le chromosome 2, ont des fréquences identiques dans les 2 échantillons. Les autres, sur le chromosome 3, sont plus abondants à Brazzaville, à l'exception de 3RC qui est l'inversion la plus fréquente de l'échantillon français. Notons, cependant, qu'aucune différence n'est significative (tabl. 2). Dans la population de Nasr'Allah, la fréquence des inversions cosmopolites du chromosome 2 ( $2 \mathrm{Lt}$ et $2 \mathrm{RNS}$ ) est supérieure à celle trouvée dans les échantillons précédents. $3 \mathrm{RC}$, par contre, est la moins représentée (situation identique à celle rencontrée à Brazzaville). La caractéristique majeure s'avère être la présence de $3 R M, 2 R C y$ ainsi que 2LCy. Ces deux dernières inversions sont responsables de la différence significative du taux d'inversion des bras $2 \mathrm{~L}$ et $2 \mathrm{R}$ entre les populations ( $p=2 \cdot 10^{-4}$ et $\mathrm{p}=8 \cdot 10^{-3}$, respectivement). Le chromosome 3 , le plus polymorphe des 2 autosomes dans les échantillons français et congolais, est celui qui présente le moins de variabilité dans la population tunisienne. Mais son taux d'inversion peut être considéré comme équivalent dans les 3 populations étudiées (tabl. 2). 


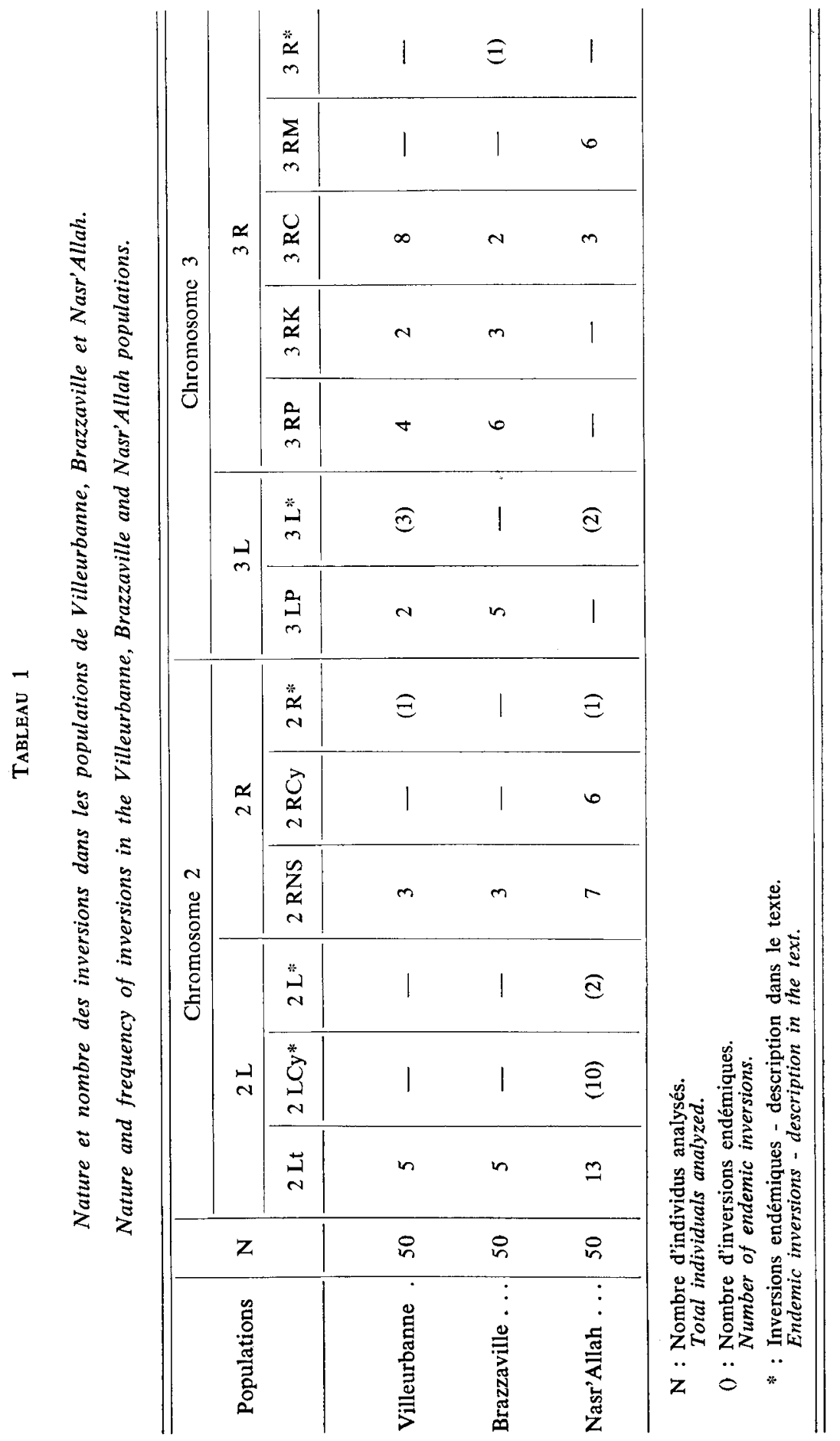




\section{TABleau 2}

Homogénéité du taux d'inversion cosmopolite et du taux d'inversion des bras chromosomiques : probabilités observées entre les échantillons de Villeurbanne (V), Brazzaville (B) et Nasr'Allah (N) pris deux à deux.

Homogeneity of cosmopolitan inversion rate and inversion rate of the major autosomes: observed probabilities between the Villeurbanne (V),

Brazzaville (B) and Nasr'Allah (N) strains two by two.

\begin{tabular}{|c|c|c|c|}
\hline & V-B & $\mathrm{N}-\mathrm{V}$ & N-B \\
\hline $2 \mathrm{Lt}$ & 0,626 & 0,04 & 0,04 \\
\hline $2 \mathrm{LCy}$ & 1 & $8.10^{-4 *}$ & $8.10^{-4 *}$ \\
\hline $2 \mathrm{~L} \ldots$ & 0,626 & $2.10^{-4 *}$ & $2.10^{-4 *}$ \\
\hline 2 RNS & 0,658 & 0,165 & 0,165 \\
\hline $2 \mathrm{RCy}$ & 1 & $0,014 *$ & $0,014 *$ \\
\hline $2 \mathrm{R} \ldots$ & 0,658 & $8.10^{-3 *}$ & $8.10^{-3 *}$ \\
\hline $3 \mathrm{LP}$ & 0,222 & 0,248 & 0,029 \\
\hline $3 \mathrm{~L}$ & 0,222 & 0,248 & 0,029 \\
\hline $3 \mathrm{RP}$ & 0,377 & 0,058 & $0,014 *$ \\
\hline $3 \mathrm{RC}$. & 0,05 & 0,107 & 0,5 \\
\hline 3 RK & 0,5 & 0,249 & 0,123 \\
\hline 3 RM & 1 & $0,014 *$ & $0,014^{*}$ \\
\hline $3 R$. & 0,335 & 0,188 & 0,407 \\
\hline
\end{tabular}

* Différences significatives - Significant differencies.

B. Distributions des inversions cosmopolites entre les individus

Chacun des 4 bras autosomaux présente soit la séquence standard ( $\mathrm{St}$ ), soit une séquence inversée (In), à l'exception du bras $3 \mathrm{~L}$ dans les lignées tunisiennes. Les individus peuvent ainsi détenir de 0 à 8 inversions, dans les échantillons de Villeurbanne et Brazzaville, de 0 à 6 dans celui de Nasr'Allah. Pour chaque bras, les homologues peuvent se combiner de la manière suivante : St/St, St/In ou In/In. Lorsqu'un bras présente plus d'un remaniement, ce qui est le cas de $3 R$ dans les 3 échantillons, $2 R$ et $2 \mathrm{~L}$ - compte tenu de $2 \mathrm{LCy}$ - dans celui de Nasr'Allah, la combinaison In/In ne représente pas des homozygotes mais correspond à la présence simultanée, sur les 2 homologues, d'un remaniement, quelle que soit sa nature. Au total, on dénombre (3) ${ }^{4}$ catégories de combinaison pour les 2 premiers échantillons, $(3)^{3}$ pour le dernier, et leur fréquence est calculée en fonction du taux d'inversion de chaque bras :

\begin{tabular}{l|l|l|l|l}
\hline \hline & $2 \mathrm{~L}$ & $2 \mathrm{R}$ & $3 \mathrm{~L}$ & $3 \mathrm{R}$ \\
\cline { 2 - 5 } Villeurbanne $\ldots \ldots \ldots$ & 0,05 & 0,03 & 0,02 & 0,14 \\
Brazzaville $\ldots \ldots \ldots$ & 0,05 & 0,03 & 0,05 & 0,11 \\
Nasr'Allah $\ldots \ldots \ldots$ & 0,23 & 0,13 & 0 & 0,09 \\
\hline \hline
\end{tabular}




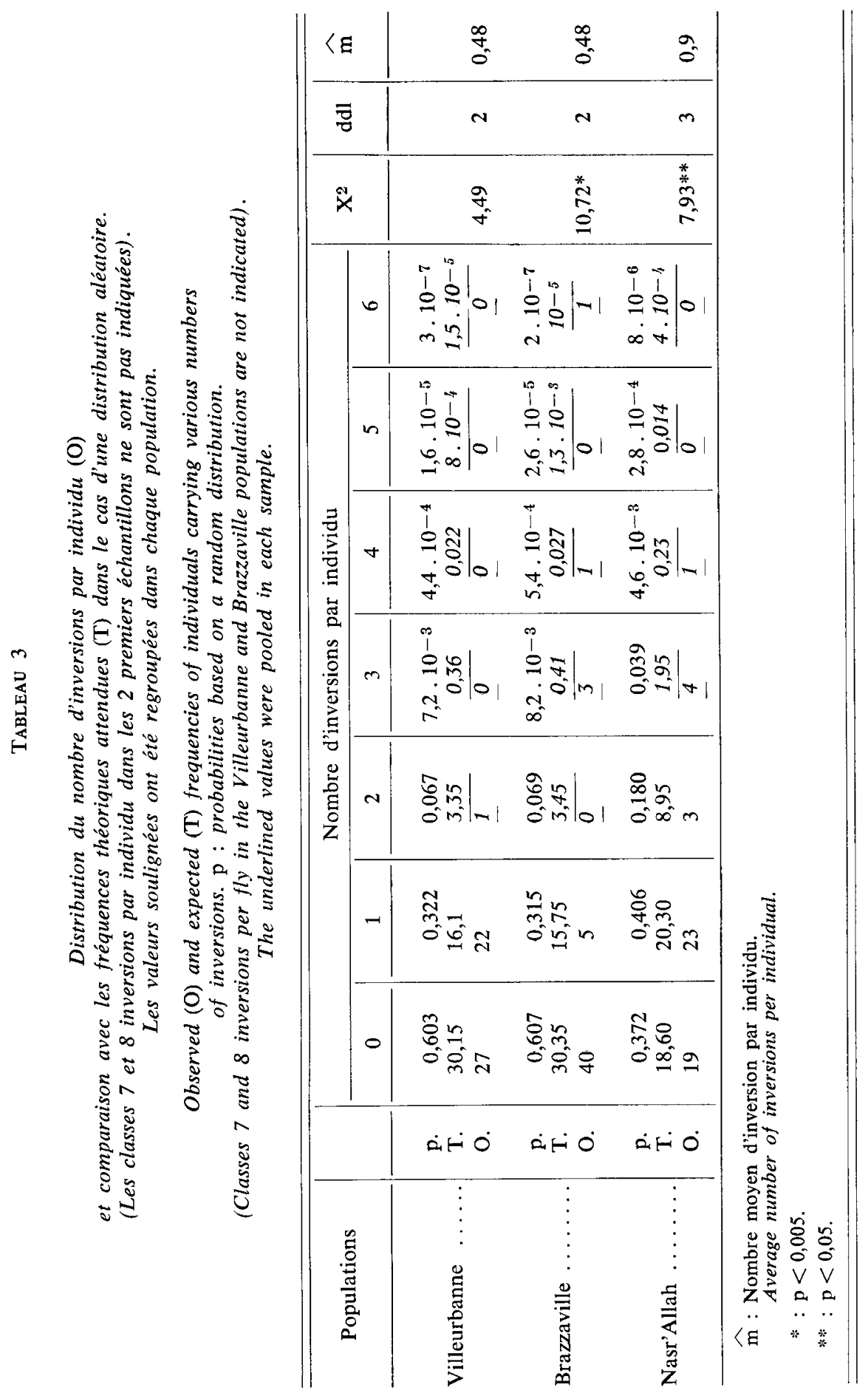


Le tableau 3 montre que les inversions se répartissent au hasard dans l'échantillon français, mais il existe cependant un excès d'individus ne détenant qu'un seul remaniement. A l'inverse, le test est hautement significatif pour la population congolaise. Celle-ci se caractérise par une distribution de type agrégatif : les inversions semblent se concentrer chez un minimum d'individus (il a été trouvé jusqu'à 6 inversions chez un seul mâle). De même, le nombre d'inversions par individu de l'échantillon tunisien n'est pas aléatoire mais leur répartition est beaucoup plus complexe que les précédentes et pourrait s'expliquer par des interactions entre différentes séquences chromosomiques.

\section{Associations intrachromosomiques}

Un autosome peut se présenter sous 4 aspects différents, avec :

a) Au moins une inversion sur chacun des deux bras (In - In).

b) Au moins une inversion sur le bras droit et aucune sur le gauche ( $\mathrm{St}-\mathrm{In}$ ).

c) Au moins une inversion sur le bras gauche et aucune sur le droit (In- St).

d) Aucune inversion, ni sur le bras gauche, ni sur le bras droit ( $\mathrm{St}-\mathrm{St}$ ).

Les remaniements sont des événements qui surviennent indépendamment les uns des autres et l'on s'attend à ce qu'ils se combinent de manière aléatoire chez les individus d'une même population. Les différentes associations entre bras d'un même chromosome sont évaluées par le coefficient de corrélation de point $R$. Les valeurs positives indiquent un excès de chromosomes présentant une inversion cosmopolite sur les deux bras simultanément et/ou de chromosomes dépourvus de tout remaniement. Les valeurs négatives signifient un déficit de ces 2 catégories.

Les probabilités d'obtenir de telles combinaisons sous l'hypothèse d'indépendance et la valeur du coefficient de corrélation pour chaque couple sont données dans le tableau 4. Lorsque l'un des bras possède plusieurs types de séquence inversée, le déséquilibre est mesuré tout d'abord en fonction de l'ensemble de ces remaniements puis pour chacun d'eux individuellement, les autres étant alors regroupés avec la séquence standard.

Dans l'échantillon de Villeurbanne, lorsque les chromosomes 2 ou 3 portent une inversion sur l'un des bras, l'autre en est dépourvu (l'unique individu détenant deux remaniements était un homozygote $2 \mathrm{Lt} / 2 \mathrm{Lt}$ ). Ceci se traduit par une valeur négative du coefficient de corrélation pour chaque couple. Cependant, malgré cette tendance générale à l'antagonisme, aucune des 5 associations prise isolément ne montre de déviation significative (tabl. 4). A l'inverse, toutes les corrélations intrachromosomiques dans les lignées congolaises sont positives et témoignent de l'excès de chromosomes des catégories a et d. Un seul déséquilibre significatif, entre les bras $3 \mathrm{~L}$ et $3 \mathrm{R}$, est mis en évidence et résulte d'une association préférentielle de $3 \mathrm{LP}$ avec $3 R C$ ( $p=0,002$ et $R=0,623$ ). Les individus de Nasr'Allah ne détiennent aucune inversion cosmopolite sur le bras $3 \mathrm{~L}$. Les associations intrachromosomiques ne sont donc testées que pour le deuxième autosome et montrent un excès de chromosomes dont les deux bras présentent simultanément des séquences inversées. Ce résultat se justifie par la combinaison non aléatoire des inversions $2 \mathrm{LCy}$ et $2 \mathrm{RCy}\left(\mathrm{p}=7 \cdot 10^{-4}\right.$ et $\left.\mathrm{R}=0,477\right)$. 


\section{TABLEAU 4}

Associations des différentes séquences chromosomiques pour chaque autosome dans les échantillons de Villeurbanne, Brazzaville et Nasr'Allah.

Probabilités obtenues sous l'hypothèse d'indépendance (p) et coefficients de corrélation de point (R).

Associations of the different chromosome sequences for each autosome in the Villeurbanne, Brazzaville and Nasr'Allah populations.

Observed probabilities based on a random combination ( $\mathrm{p})$ and correlation coefficients $(\mathbf{R})$.

\begin{tabular}{l|l|l|l|l|l}
\hline \hline $\begin{array}{c}\text { Associations } \\
\text { chromosomiques }\end{array}$ & \multicolumn{3}{|c|}{ Type de chromosomes*** } & p & R \\
\cline { 2 - 4 } & $\mathrm{a}$ & $\mathrm{b}$ & $\mathrm{c}$ & $\mathrm{d}$ & \\
\end{tabular}

\begin{tabular}{ll|r|r|r|r|r}
2 Lt-2 RNS $\ldots \ldots \ldots$ & 0 & 3 & 5 & 92 & 0,856 & $-0,040$ \\
3 LP-3 R** $\ldots \ldots \ldots$ & 0 & 14 & 2 & 84 & 0,738 & $-0,058$ \\
3 LP-3 RP $\ldots \ldots \ldots$ & 0 & 4 & 2 & 94 & 0,921 & $-0,029$ \\
3 LP-3 RC $\ldots \ldots \ldots$ & 0 & 8 & 2 & 90 & 0,846 & $-0,042$ \\
3 LP-3 RK $\ldots \ldots \ldots$ & 0 & 2 & 2 & 96 & 0,960 & $-0,020$
\end{tabular}

II. Brazzaville

\begin{tabular}{l|l|l|l|l|l|l}
2 Lt-2 RNS $\ldots \ldots \ldots$ & 1 & 2 & 4 & 93 & 0,994 & 0,228 \\
3 LP-3 R** $\ldots \ldots \ldots$ & 5 & 6 & 0 & 89 & $6.10^{-6 *}$ & 0,652 \\
3 LP-3 RP $\ldots \ldots \ldots$ & 2 & 4 & 3 & 91 & 0,999 & 0,328 \\
3 LP-3 RC $\ldots \ldots \ldots$ & 2 & 0 & 3 & 95 & $0,002^{*}$ & 0,623 \\
3 LP-3 RK $\ldots \ldots \ldots$ & 1 & 2 & 4 & 93 & 0,994 & 0,229
\end{tabular}

III. Nasr'Allah

\begin{tabular}{ll|l|l|l|l|l|r}
2 L**-2 R** $\ldots \ldots$ & 7 & 6 & 16 & 71 & $0,012 *$ & 0,283 \\
2 Lt-2 RNS $\ldots \ldots \ldots$ & 1 & 6 & 12 & 81 & 0,775 & 0,010 \\
2 LCy-2 RNS $\ldots \ldots$ & 0 & 7 & 10 & 83 & 0,467 & $-0,091$ \\
2 Lt-2 RCy $\ldots \ldots \ldots$ & 2 & 4 & 11 & 83 & 0,972 & 0,153 \\
2 LCy-2 RCy $\ldots \ldots$ & 4 & 2 & 6 & 88 & $7.10^{-4 *}$ & 0,477 \\
\hline
\end{tabular}

*: Déséquilibre significatif.

Significant disequilibrium.

$* *:$ Ensemble des inversions.

All inversions.

*** : (a) chromosome de type In-In, (b) chromosome St-In, (c) chromosome In-St, (d) chromosome St-St. 


\section{Associations interchromosomiques}

Quatre classes gamétiques sont susceptibles de se rencontrer, avec :

a) Au moins une inversion sur chacun des 2 autosomes (2In - 3In).

b) Au moins une inversion sur le chromosome 3 et aucune sur le 2 (2St $-3 \mathrm{In}$ ).

c) Au moins une inversion sur le chromosome 2 et aucune sur le 3 ( $2 \mathrm{In}-3 \mathrm{St}$ ).

d) Aucune inversion sur les 2 autosomes ( $2 \mathrm{St}-3 \mathrm{St}$ ).

La question se pose de savoir s'il existe des associations particulières entre les 2 autosomes révélées par un excès de l'une ou l'autre de ces catégories de gamète. De telles associations pourraient résulter d'une sélection différentielle des génotypes.

Dans l'échantillon de Villeurbanne, les valeurs du coefficient de corrélation $\mathbf{R}$ sont toutes négatives (tabl. 5). Le déséquilibre constaté entre les 2 autosomes ( $p=0,004$ et $\mathbf{R}=-0,136$ ) ne résulte d'aucun antagonisme entre inversions particulières mais de lensemble des remaniements du chromosome 2 et de ceux du bras $3 R$. Un déséquilibre significatif est également détecté au sein des gamètes de la population congolaise $\left(\mathrm{p}=4 \cdot 10^{-9}\right.$ et $\left.\mathrm{R}=0,425\right)$. Il est produit par un excès d'associations de $2 \mathrm{Lt}$ avec l'ensemble des inversions du bras $3 R$, d'une part et 2RNS-3RK d'autre part ( $p=10^{-4}$, $R=0,289$ et $p=4 \cdot 10^{-8}, R=0,660$ respectivement). Les combinaisons des séquences inversées et standard des 2 autosomes sont aléatoires dans l'échantillon tunisien. Cependant, les inversions $2 \mathrm{LCy}, 2 \mathrm{RNS}$ et $3 \mathrm{RC}$ se caractérisent, vis-à-vis des remaniements portés par l'autre chromosome, par des corrélations négatives systématiques. L'excès d'association n'est significatif qu'entre $3 \mathrm{RC}$ et la séquence standard du chromosome 2 .

L'origine d'un tel déséquilibre peut être multiple. Pour l'expliquer, il est nécessaire de rappeler les différentes étapes suivies au cours du protocole expérimental : récolte de femelles inséminées de la nature, constitution de lignées isofemelles, prélèvement d'un mâle de chaque lignée, lequel est ensuite croisé avec une femelle de référence, analyse d'un certain nombre de gamètes du mâle par l'intermédiaire des larves $F 1$ de troisième stade larvaire. Le déséquilibre mis en évidence au sein des gamètes peut être engendré par :

1) l'existence de combinaisons non aléatoires des autosomes chez les mâles, donc par un déséquilibre des génotypes ;

2) le tirage non aléatoire des gamètes analysés, en raison : étudiés ;

- d'un effet d'échantillonnage des larves F 1, c'est-à-dire des gamètes des 50 mâles

- de la mortalité de certains descendants entre la fécondation et le troisième stade larvaire au cours duquel se fait l'analyse cytogénétique ;

- d'une ségrégation non aléatoire des autosomes au cours de la spermatogénèse des mâles.

Si le tirage des gamètes n'est pas aléatoire, les fréquences observées de leur génotype et celles attendues (calculées en fonction du génotype des mâles et du nombre de leurs gamètes étuđiés) peuvent révéler des différences significatives.

Le nombre de génotypes différents attendus pour les gamètes des trois échantillons est de :

7 pour Villeurbanne, avec 337 gamètes analysés;

14 pour Brazzaville, avec 322 gamètes analysés;

13 pour Nasr'Allah, avec 316 gamètes analysés. 


\section{Tableau 5}

Associations interchromosomiques dans les échantillons de Villeurbanne, Brazzaville et Nasr'Allah.

Probabilités obtenues sous l'hypothèse d'indépendance ( $\mathrm{p}$ ) et coefficients de corrélation de point (R).

Associations between chromosomes 2 and 3

in the Villeurbanne, Brazzaville, and Nasr'Allah populations.

Observed probabilities based on a random combination $(\mathrm{p})$ and correlation coefficients $(\mathrm{R})$.

\begin{tabular}{l|c|c|c|c|c|}
\hline \hline $\begin{array}{c}\text { Associations } \\
\text { chromosomiques }\end{array}$ & \multicolumn{3}{|c|}{ Type de gamètes *** } & $\mathrm{p}$ & $\mathrm{R}$ \\
\hline
\end{tabular}

I. Villeurbanne

\begin{tabular}{|c|c|c|c|c|c|c|}
\hline$\ldots \ldots \ldots$ & 0 & 59 & 27 & 251 & $0,004 *$ & $\longrightarrow 0,136$ \\
\hline $2 * *-3 \mathrm{LP}$ & 0 & 9 & 27 & 301 & 0,467 & $-0,049$ \\
\hline $2 * *-3 \mathbf{R} * *$ & 0 & 50 & 27 & 260 & $0,011^{*}$ & $-0,123$ \\
\hline $2 * *-3 \mathrm{RP}$ & 0 & 14 & 27 & 296 & 0,303 & $-0,061$ \\
\hline $2 * *-3 \mathrm{RK}$ & 0 & 8 & 27 & 302 & 0,504 & $-0,046$ \\
\hline $2 * *-3 \mathrm{RC}$ & 0 & 28 & 27 & 282 & 0,078 & $-0,089$ \\
\hline $2 \mathrm{Lt}-3 * *$. & 0 & 59 & 16 & 262 & 0,042 & $-0,103$ \\
\hline $2 \mathrm{Lt}-3 \mathrm{LP}$ & 0 & 9 & 16 & 312 & 0,640 & $-0,037$ \\
\hline $2 \mathrm{Lt}-3 \mathrm{R} * *$ & 0 & 50 & 16 & 271 & 0,070 & $-0,093$ \\
\hline $2 \mathrm{Lt}-3$ RP $\ldots \ldots \ldots$ & 0 & 14 & 16 & 307 & 0,499 & $-0,046$ \\
\hline 2 Lt-3 RK & 0 & 8 & 16 & 313 & 0,675 & $-0,035$ \\
\hline 2 Lt-3 RC & 0 & 28 & 16 & 293 & 0,241 & $-0,070$ \\
\hline 2 RNS-3** & 0 & 59 & 11 & 267 & 0,116 & $-0,084$ \\
\hline 2 RNS-3 LP $\ldots \ldots \ldots$ & 0 & 9 & 11 & 317 & 0,739 & $-0,030$ \\
\hline 2 RNS-3 R** & $\mathbf{0}$ & 50 & 11 & 276 & 0,166 & 一 0,077 \\
\hline 2 RNS-3 RP $\ldots \ldots \ldots$ & 0 & 14 & 11 & 312 & 0,622 & $-0,038$ \\
\hline 2 RNS-3 RK $\ldots \ldots \ldots$ & $\mathbf{0}$ & 8 & 11 & 318 & 0,765 & $-0,029$ \\
\hline 2 RNS-3 RC $\ldots \ldots$. & $\mathbf{0}$ & 28 & 11 & 298 & 0,379 & $-0,055$ \\
\hline
\end{tabular}

\section{Brazzaville}

\begin{tabular}{|c|c|c|c|c|c|c|}
\hline $2 * *-3 * *$ & 13 & 27 & 6 & 276 & $4.10-9 *$ & 0,425 \\
\hline$\ldots \ldots \ldots \ldots$ & 5 & 15 & 14 & 288 & 0,999 & 0,208 \\
\hline $2 * *-3 \mathrm{R} * * \ldots \ldots \ldots$ & 13 & 27 & 6 & 276 & $4.10^{-9 *}$ & 0,425 \\
\hline $2 * *-3$ RP $\ldots \ldots \ldots$ & 4 & 20 & 15 & 283 & 0,991 & 0,130 \\
\hline $2 * *-3$ RK $\ldots \ldots \ldots$ & 5 & 3 & 14 & 300 & $2.10^{-5 *}$ & 0,383 \\
\hline $2 * *-3$ RC $\ldots \ldots \ldots$ & 4 & 8 & 15 & 295 & 0,999 & 0,299 \\
\hline $2 \mathrm{Lt}-3^{* *} \ldots \ldots \ldots \ldots$ & 8 & 36 & 6 & 272 & $10^{-4 *}$ & 0,289 \\
\hline $2 \mathrm{Lt}-3 \mathrm{LP}$ & 4 & 16 & 10 & 292 & 0,999 & 0,197 \\
\hline $2 \mathrm{Lt}-3 \mathrm{R}^{* *} \ldots \ldots \ldots$ & 8 & 36 & 6 & 272 & $10^{-4 *}$ & 0,289 \\
\hline 2 Lt-3 RP $\ldots \ldots \ldots \ldots$ & 4 & 20 & 10 & 288 & 0,998 & 0,171 \\
\hline 2 Lt-3 RK $\ldots \ldots \ldots$ & 0 & 8 & 14 & 300 & 0,698 & $-0,034$ \\
\hline $2 \mathrm{Lt}-3 \mathrm{RC} \ldots \ldots \ldots$ & 4 & 4 & 10 & 304 & 0,999 & 0,357 \\
\hline 2 RNS-3** $\ldots \ldots \ldots$ & 7 & 33 & 0 & 282 & $3.10^{-7 *}$ & 0,396 \\
\hline 2 RNS-3 LP . . . . . . & 3 & 17 & 4 & 298 & 0,999 & 0,226 \\
\hline 2 RNS-3 R** $\ldots \ldots$ & 7 & 33 & $\mathbf{0}$ & 282 & $3.10^{-7 *}$ & 0,396 \\
\hline 2 RNS-3 RP. & 0 & 24 & 7 & 291 & 0,578 & $-0,042$ \\
\hline 2 RNS-3 RK $\ldots$ & 5 & 3 & 2 & 312 & $4.10^{-8 *}$ & 0,660 \\
\hline 2 RNS-3 RC & 2 & 6 & 5 & 309 & 0,999 & 0,250 \\
\hline
\end{tabular}


TABLEAU 5 (suite)

\begin{tabular}{c|c|c|c|}
$\begin{array}{c}\text { Associations } \\
\text { chromosomiques }\end{array}$ & $\mathbf{p}$ & $\mathbf{d}$ \\
\hline & $\mathbf{a}$
\end{tabular}

III. Nasr'Allah

\begin{tabular}{|c|c|c|c|c|c|c|}
\hline $2 * *-3 * * \quad \ldots \ldots \ldots \ldots$ & 8 & 24 & 90 & 194 & 0,288 & $-0,044$ \\
\hline $2 * *-3 \mathbf{R} * * \ldots \ldots \ldots$ & 8 & 24 & 90 & 194 & 0,288 & $-0,044$ \\
\hline $2 * *-3 \mathrm{RC} \ldots \ldots \ldots$ & 0 & 10 & 98 & 208 & $0,019 *$ & $-0,121$ \\
\hline $2 * *-3$ RM $\ldots \ldots \ldots$ & 8 & 14 & 90 & 204 & 0,791 & 0,032 \\
\hline $2 \mathrm{~L}^{* *}-3 \mathrm{R}^{* *} \ldots \ldots$ & 8 & 24 & 68 & 216 & 0,647 & 0,007 \\
\hline $2 \mathrm{~L} * *-3 \mathrm{RC} \ldots \ldots \ldots$ & 0 & 10 & 76 & 230 & 0,061 & $-0,102$ \\
\hline $2 \mathrm{~L}^{* *}-3$ RM $\ldots \ldots \ldots$ & 8 & 14 & 68 & 226 & 0,946 & 0,079 \\
\hline $2 \mathrm{Lt}-3 \mathrm{R} * * \quad \ldots \ldots \ldots$ & 8 & 24 & 50 & 234 & 0,894 & 0,058 \\
\hline $2 \mathrm{Lt}-3 \mathrm{RC} \quad \ldots \ldots \ldots$ & 0 & 10 & 58 & 248 & 0,127 & $-0,086$ \\
\hline 2 Lt-3 RM . . . . . . & 8 & 14 & 50 & 244 & 0,991 & 0,127 \\
\hline $2 \mathrm{LCy}-3 \mathbf{R} * * \ldots \ldots$ & 0 & 32 & 18 & 266 & 0,138 & $-0,082$ \\
\hline $2 \mathrm{LCy}-3 \mathrm{RC} \ldots \ldots$ & 0 & 10 & 18 & 288 & 0,551 & $-0,044$ \\
\hline 2 LCy-3 RM . . . . . & 0 & 22 & 18 & 276 & 0,263 & $-0,067$ \\
\hline $2 \mathrm{R}^{* *-3} \mathrm{R} * * \ldots \ldots$ & 7 & 25 & 36 & 248 & 0,949 & 0,081 \\
\hline $2 \mathbf{R}^{* *-3} \mathrm{RC} \ldots \ldots$ & 0 & 10 & 43 & 263 & 0,226 & $-0,072$ \\
\hline $2 \mathbf{R} * *-3 \mathbf{R M} \ldots \ldots$ & 7 & 15 & 36 & 258 & 0,995 & 0,145 \\
\hline 2 RNS-3 R** ..... & 0 & 32 & 24 & 260 & 0,069 & $-0,096$ \\
\hline 2 RNS-3 RC ....... & 0 & 10 & 24 & 282 & 0,448 & $\longrightarrow 0,052$ \\
\hline 2 RNS-3 RM . . . . . & 0 & 22 & 24 & 270 & 0,165 & $-0,078$ \\
\hline 2 RCy-3 R** $\ldots \ldots$ & 7 & 25 & 12 & 272 & 0,999 & 0,224 \\
\hline $2 \mathrm{RCy}-3 \mathrm{RC} \ldots \ldots$ & 0 & 10 & 19 & 287 & 0,553 & $-0,046$ \\
\hline 2 RCy-3 RM ...... & 7 & 15 & 12 & 282 & 0,999 & 0,297 \\
\hline
\end{tabular}

* : Associations non aléatoires.

Significant non-random association.

** : Ensemble des inversions. All inversions.

*** : (a) combinaison In-In, (b) combinaison St-In, (c) combinaison In-St, (d) combinaison St-St.

Les différences entre distributions observées et distributions théoriques ne sont significatives dans aucun des échantillons :

Villeurbanne $\mathrm{X}^{2}=1,01-6 \mathrm{ddl}-\mathrm{p}>0,05$;

Brazzaville $\quad \mathrm{X}^{2}=13,6-13 \mathrm{ddl}-\mathrm{p}>0,05$;

Nasr'Allah $\quad X^{2}=9,05-12 \mathrm{ddl}-\mathrm{p}>0,05$.

Nous en concluons donc, au risque de 5 p. 100, que les gamètes analysés sont conformes aux génotypes des mâles. L'existence des combinaisons non aléatoires des autosomes au sein des gamètes nous semble donc refléter le déséquilibre des génotypes des mâles étudiés. 


\section{Discussion et conclusion}

Les résultats obtenus montrent que, dans chacune des populations étudiées, les inversions ne sont pas distribuées au hasard, selon le modèle panmictique d'une population infinie. En fait, 2 catégories de phénomènes ont été mises en évidence : d'une part des associations entre certaines séquences portées par le même chromosome, c'est-à-dire un déséquilibre de liaison entre inversions liées physiquement, d'autre part des associations interchromosomiques, entre les chromosomes 2 et 3 . Or, quand plusieurs inversions «indépendantes » existent sur le même chromosome et à plus forte raison sur des chromosomes différents, on s'attend à ce qu'elles se combinent au hasard chez les individus d'une même population. Les 2 phénomènes observés relevant de mécanismes essentiellement différents, il importe de les discuter séparément.

En ce qui concerne les inversions liées, nous pouvons remarquer qu'elles affectent toujours des bras chromosomiques différents. Ainsi, dans la population de Villeurbanne, il existe un antagonisme systématique, bien que non significatif, entre tous les remaniements. Nous avons obtenu, dans l'échantillon de Brazzaville, un excès de chromosomes 3 portant simultanément une inversion sur chaque bras et plus particulièrement les séquences $3 \mathrm{LP}$ et $3 \mathrm{RC}$. Dans la population tunisienne, la plus polymorphe des trois, les interactions sont plus complexes en raison des tendances à l'association pour certaines inversions, à l'antagonisme pour d'autres. Divers mécanismes peuvent expliquer un déséquilibre de liaison. Théoriquement, plus la séquence standard entre deux remaniements est faible, plus les recombinaisons entre eux sont rares. De plus, on sait que chez un hétérozygote la suppression des recombinaisons concerne non seulement les segments inversés, mais aussi les régions proches des points de cassure en raison du mauvais appariement des homologues au cours de la méiose (DobZhansKy \& Epling, 1948). Deux inversions mettront donc d'autant plus de temps à se séparer qu'elles sont plus proches l'une de l'autre. Par ailleurs, la présence du centromère et de l'hétérochromatine juxtacentromérique entre ces inversions réduit également la recombinaison (il semble en effet que les remaniements qui surviennent dans cette région soient éliminés des populations naturelles de $D$. melanogaster). La faible fréquence des recombinaisons paraît pouvoir être invoquée dans le cas de la population de Nasr'Allah où l'on trouve la séquence $2 \mathrm{RCy}$ : celle-ci n'existe en effet qu'en présence d'une inversion du bras gauche, 2Lt ou 2LCy, mais le seul déséquilibre significatif concerne le couple 2LCy$2 \mathrm{RCy}$, bien que la distance qui sépare ces remaniements soit pratiquement identique (fig. 1). On peut supposer que l'inversion $2 \mathrm{RCy}$ s'est formée sur un chromosome portant déjà $2 \mathrm{LCy}$. Le chromosome $2 \mathrm{Lt}-2 \mathrm{RCy}$ serait apparu plus récemment à la suite d'une rare recombinaison chez un hétérozygote $2 \mathrm{LCy}-2 \mathrm{RCy} / 2 \mathrm{Lt}-2 \mathrm{RSt}$. La non observation de 2LSt-2RCy peut provenir d'un nombre insuffisant d'individus analysés. De la même façon, ceci expliquerait l'excès de chromosomes $3 \mathrm{LP}-3 \mathrm{RC}$ du Congo puisqu'un tel déséquilibre n'est pas mis en évidence avec $3 R P$ et $3 R K$ qui offrent cependant moins de possibilités de recombinaison.

Ainsi, en présence d'une association préférentielle entre 2 structures génétiques physiquement liées, la première explication consiste à faire intervenir le hasard et les événements historiques. Il peut s'agir d'une apparition récente de l'inversion considérée, ou bien d'une population qui, à la suite d'accidents divers (baisse des effectifs, introduction de migrants) n'a pas encore récupéré son équilibre. Mais une interprétation tout-à-fait opposée, faisant intervenir la sélection naturelle est aussi possible, si l'on 
admet que les diverses associations ont des valeurs adaptatives différentes. De nombreux exemples de déséquilibre de liaison entre inversions ont déjà été signalés chez diverses espèces de Drosophiles : D. robusta (Levitan, 1955, 1958, 1961, 1973 a, 1973 b, 1978 ; Etges, 1984), D. guaramunu (Levitan \& Salzano, 1959), D. paramelanica et D. euronotus (STAlKeR, 1960, 1964), D. rubida (MATHER, 1961, 1963), D. subobscura (Krimbas, 1964 ; Krimbas \& Zouros, 1969 ; Zouros et al., 1974 ; Sperlich \& Feuerbach-Mravlag, 1974 ; Krimbas \& Loukas, 1980). Chez ces espèces, les auteurs ont généralement invoqué le maintien du déséquilibre de liaison par la sélection naturelle. Chez $D$. robusta par exemple, des variations saisonnières et altitudinales de la fréquence des associations ont été décrites (LEvitan, 1973 a, 1973 b, 1978 ; ETgEs, 1984). Chez $D$. melanogaster, des associations non aléatoires ont été signalées plus récemment dans diverses populations naturelles, en Grèce (Alahiotis et al., 1976), aux Etats-Unis (LANGley et al., 1977), au Japon (INOUE \& Watanabe, 1979) et en Australie (KNIBB et al., 1981). D’une manière générale, le déséquilibre se caractérise par un excès de chromosomes dont les 2 bras possèdent des inversions. Une façon d'étudier la signification de ces associations préférentielles serait de voir si elles se maintiennent dans la même population au cours des années successives.

Les combinaisons non aléatoires entre les 2 autosomes constituent une autre observation intéressante. Nous avons vu que ces associations ne semblaient pas pouvoir s'expliquer par des anomalies de ségrégation et donc qu'elles reflétaient très probablement les fréquences initiales observées chez les individus sauvages ayant servi à fonder les lignées isofemelles. Une difficulté importante pour l'interprétation des observations réside dans le fait que nous ne savons pas comment la fréquence des inversions évolue au sein de telles lignées conservées au laboratoire. On sait que dans des populations japonaises en élevage de masse, les inversions sont désavantagées et progressivement éliminées en un ou deux ans (InOue, 1979). La méthode des lignées isofemelles est certainement bien meilleure que l'élevage de masse pour conserver la diversité génétique. Par ailleurs, un délai assez faible s'est écoulé entre la collecte du matériel et son étude. Notons enfin qu'il n'est pas du tout certain que le désavantage observé par INOUE existe pour toutes les populations de la planète lorsqu'elles sont conservées au laboratoire. Nous avons quelques indications de la très longue conservation des inversions dans des lignées africaines maintenues depuis plus de deux ans (LEMEUNIER, données non publiées). Nous pouvons donc raisonnablement admettre que les résultats obtenus concernant aussi bien les fréquences globales des inversions que les associations préférentielles reflètent la structure génétique existant dans les populations naturelles considérées. En dépit de l'identité du protocole expérimental et des conditions d'élevage, les résultats sont très différents selon les populations étudiées. L'opposition est frappante entre l'antagonisme général des remaniements dans la population française et, au contraire, leur concentration chez les individus de Brazzaville. Si des associations de ce type ont été signalées depuis longtemps chez Moraba scurra (LEWONTIN \& WHITE, 1960), très peu sont connues chez les Drosophiles. En fait, les associations observées concernent toutes D. melanogaster, en Amérique (STAlKer, 1976) ou en Australie (KNiBB et al., 1981). Les résultats de STALKER font état de corrélations négatives entre les deux autosomes. Cette observation s'est trouvée corroborée par KNIBB et al. pour les populations dont le nombre moyen d'inversions par individu était supérieur à un. A l'inverse, les populations de latitudes plus élevées, qui avaient moins d'un remaniement par mouche, présentaient une association positive. Les résultats obtenus dans ce travail ne reproduisent pas les observations de KNIBB et al. Les populations de Brazzaville et Villeurbanne ont toutes les deux un nombre moyen d'inversions par individu inférieur à un. Or, dans le premier cas, une nette distribution agrégative a été observée en raison des corrélations positives. 
Et, dans le deuxième cas, bien que la population française soit de latitude élevée, la distribution est au contraire trop uniforme à cause des corrélations négatives entre les inversions. Des événements historiques ne peuvent être invoqués pour expliquer des associations interchromosomiques non aléatoires en raison du brassage des chromosomes lors de chaque méiose. Les phénomènes sélectifs pourraient justifier ces déséquilibres. Chez les femelles hétérozygotes, l'élimination des gamètes recombinés, lorsqu'un crossing-over survient au niveau d'une inversion, a pour effet de diminuer le nombre de remaniements présents chez le même individu. Mais le phénomène paraît de faible importance et l'on ne sait pas si, chez D. melanogaster, les chromatides recombinées ne sont pas incorporées dans les globules polaires comme l'a montré CARson (1946) chez Sciara impatiens.

Le dernier point qui mérite d'être souligné est la très grande similitude des échantillons français et congolais de par la nature et la fréquence des inversions cosmopolites. Les études effectuées en Amérique (STAlKer, 1976 ; Mettler et al., 1977), au Japon (Watanabe, 1967 ; Inoue \& Watanabe, 1979) et en Australie (KNIBB et al., 1981) ont montré une répartition des inversions selon des clines latitudinaux. D'une façon générale, les régions tempérées sont pauvres en inversions et la diversité augmente lorsqu'on se rapproche de l'équateur. La population tempérée de Villeurbanne correspond bien à ce schéma général, en revanche on s'attendait à trouver un très fort polymorphisme dans la population de Brazzaville, et ceci d'autant plus que la région Afrotropicale abrite des populations proches de l'état ancestral (TsaCas \& LaCHaISE, 1974 ; TsaCAS, 1979 ; David, 1982). Or, la population de Brazzaville, même si elle présente certaines caractéristiques particulières, est très peu polymorphe par rapport aux populations subtropicales étudiées en Australie ou en Amérique. Des études sur d'autres populations africaines sont souhaitables avant que l'on puisse cherchér une interprétation des observations présentées ici.

Reçu le 24 décembre 1984.
Accepté le 13 mars 1985.

\section{Remerciements}

Les auteurs expriment leur gratitude au Docteur J.R. DAvid pour ses suggestions et remarques sur la rédaction de ce travail. Le Docteur M. Ashburner nous a confirmé l'interprétation de certaines inversions et nous tenons à l'en remercier.

Les lignées de Villeurbanne nous ont été fournies par le Docteur J.R. DAvid, celles du Congo par le Docteur J. Vourdibio, de la Faculté des Sciences de Brazzaville (Congo) et nous désirons les en remercier ainsi que les autorités tunisiennes qui nous ont permis de récolter les lignées de Nasr'Allah.

\section{Références bibliographiques}

Alahiotis S., Pelecanos M., Zacharopoulou A., 1976. A contribution to the study of linkage disequilibrium in D. melanogaster. Canad. J. Genet. Cytol., 18, 739-745.

Ashburner M., 1967. Patterns of puffing activity in the salivary gland chromosomes of Drosophila. I. Autosomal puffing patterns in a laboratory stock of $D$. melanogaster. Chromosoma, 21, 398-428. 
Ashburner M., Lemeunier F., 1976. Relationships within the melanogaster species subgroup of the genus Drosophila (Sophophora). I. Inversion polymorphisms in D. melanogaster and D. simulans. Proc. R. Soc. Lond. B., 193, 137-157.

BRIDGES C.B., 1935. Salivary chromosome maps. J. Hered., 26, 60-64.

Carson H.L., 1946. The selective elimination of inversion dicentric chromatids during meiosis in the eggs of Sciara impatiens. Genetics, 31, 95-113.

Dagnelie P., 1973. Théorie et méthodes statistiques. 2 Vol., $2^{\mathrm{e}}$ éd., Les presses agronomiques de Gembloux, A.S.B.L. Editions J. Duculot, S.A. Gembloux.

DAVID J.R., 1982. Latitudinal variability of Drosophila melanogaster : Allozyme frequencies divergence between European and Afrotropical populations. Biochem. Genet., 20, 747-761.

Dobzhansky Th., Epling C., 1948. The suppression of crossing-over in inversion heterozygotes of D. pseudoobscura. Proc. Nat. Acad. Sci. U.S., 34, 137-141.

ETGES W.J., 1984. Genetic structure and change in natural populations of $D$. robusta : systematic inversion and inversion association frequency shifts in the Great Smoky Mountains. Evolution, 38, 675-688.

EVERITT B.S., 1977. Monographs on applied probability and statistics. The analysis of contingency tables. 128 pp., Chapman and Hall, London.

INOUE Y., 1979 a. The fate of polymorphic inversions of Drosophila melanogaster transferred to laboratory conditions. Japan. J. Genetics, 54, 83-96.

InOue Y., 1979 b. Seasonal changes of inversions frequencies of D. melanogaster. Ann. Rept. Nat. Inst. Genetics, Japan, 29, 77

INOUE Y., WATANABE K., 1979. Inversion polymorphisms in Japanese natural populations of Drosophila melanogaster. Japan. J. Genetics., 54, 69-82.

KNIBB W.R., 1982. Chromosome inversion polymorphisms in Drosophila melanogaster II. Geographic clines and climatic associations in Australasia, North America and Asia. Genetica, 58, 213-221

KnibB W.R., OAKeshott J.G., Gibson J.B., 1981. Chromosome inversion polymorphism in D. melanogaster. I. Latitudinal clines and association between inversions in Australian populations. Genetics, 98, 833-847.

Krimbas C.B., 1964. The genetics of $D$. subobscura populations. I. Inversion polymorphism in populations of Southern Greece. Evolution, 18, 541-552.

Krimbas C.B., Loukas M., 1980. The inversion polymorphism of Drosophila subobscura. In : Hecht M.K., STeere W.C., Wallace B. (ed.), Evolutionary Biology, Vol. 12, Plenum Press.

Krimbas C.B., Zouros E., 1969. Crossing-over suppression between linked but non-overlapping inversions of D. subobscura. D.I.S., 47, 98.

Langley C.H., Ito K., VoelKer R.A., 1977. Linkage disequilibrium in natural populations of Drosophila melanogaster. Seasonal variation. Genetics, 86, 447-454.

LEFEVRE G., 1976. A photographic representation and interpretation of the polytene chromosomes of Drosophila melanogaster salivary glands. In : AsHBURNER M., NoVITSKY E. (ed.), The Genetics and Biology of Drosophila, Vol. 1 a, Academic Press, London.

Lefort G., 1967. Mathématiques pour les Sciences Biologiques et Agronomiques. 680 pp., Collection U, Armand Collin, Paris.

Lemeunier F., David J., Tsacas L., Ashburner M., 1985. The melanogaster species group. In : Ashburner M., Carson H.L., Thompson J.N. (ed.), The Genetics and Biology of Drosophila, Vol. 3, Academic Press, London.

Levitan M., 1955. Studies of linkage in populations. I. Association of second chromosome linkages in Drosophila robusta. Evolution, 9, 62-74.

LeVitan M., 1958. Non-random associations of inversions. Cold Spring Harbor Symposia on Quantitative Biology, 23, 251-268.

Levitan M., 1961. Proof of an adaptative linkage association. Science, 134, 1617-1619.

Levitan M., 1973 a. Studies of linkage in populations. VI. Periodic selection for X-Chromosome gene arrangement combinations. Evolution, 27, 215-225. 
Levitan M., $1973 \mathrm{~b}$. Studies of linkage in populations. VII. Temporal variation and X-Chromosomal linkage disequilibriums. Evolution, 27, 476-485.

LeVITAN M., 1978. Studies of linkage in populations. IX. The effect of altitude on X-Chromosomal arrangement combinations in D. robusta. Genetics, 89, 751-763.

LeVitan M., Salzano F.N., 1959. Studies of linkage in populations. III. An association of linked inversions in Drosophila guaramunu. Heredity, 13, 243-248.

LEWONTIN R.C., WHTE M.J.D., 1960. Interaction between inversion polymorphisms of two chromosome pairs in the grasshopper Moraba scurra. Evolution, 14, 116-129.

Mather W.B., 1961. Chromosomal polymorphism in Drosophila rubida Mather. Genetics, 46, 799-810.

MATHER W.B., 1963. Ecological and sexual variation in D. rubida inversion polymorphism. Heredity, 18, 109-111.

Mettler L.E., VoelKer R.A., MukaI T., 1977. Inversion clines in populations of Drosophila melanogaster. Genetics, 87, 169-176.

SIEGEL S., 1956. Non parametric statistics for the behavioral sciences. 312 pp., Mac Graw Hill, New York.

Sperlich D., Feuerbach-Mravlag H., 1974. Epistatic gene interaction, crossing-over and linked and unlinked inversions in Drosophila subobscura. Evolution, 28, 67-75.

Stalker H.D., 1960. Chromosomal polymorphism in Drosophila paramelanica Patterson. Genetics, 45, 95-114.

Stalker H.D., 1964. Chromosomal polymorphism in Drosophila euronotus. Genetics, 49, 669-687.

StalKeR H.D., 1976. Chromosome studies in wild populations of Drosophila melanogaster. Genetics, 82, 323-347.

Stalker H.D., 1980. Chromosome studies in wild populations of D. melanogaster. II. Relationship of inversion frequencies to latitude, season, wing-loading and flight activity. Genetics, 95, 211-223.

TSACAS L., 1979. Contribution des données africaines à la compréhension de la biogéographie et de l'évolution du sous-genre Drosophila (Sophophora) Sturtevant (Diptera, Drosophilidae). C.R. Soc. Biogéogr., 480, 29-51.

TsaCas L., Lachaise D., 1974. Quatre nouvelles espèces de la Côte-d'Ivoire du genre Drosophila, groupe melanogaster, et discussion de l'origine du sous-groupe melanogaster (Diptera : Drosophilidae). Ann. Univ. Abidjan, Série E (Ecologie), Tome VII, fasc. 1, 193-211.

Watanabe T.K., 1969. Persistence of visible mutant in natural populations of Drosophila melanogaster. Japan. J. Genetics, 44, 15-22.

Watanabe T.K., Watanabe T., 1973. Fertility genes in natural populations of Drosophila melanogaster. III. Superiority of inversion heterozygotes. Evolution, 27, 468-475.

Watanabe T.K., Watanabe T., 1977. Enzyme and chromosome polymorphisms in Japanese natural populations of Drosophila melanogaster. Genetics, 85, 319-329.

Watanabe T.K., Watanabe T., Oshima C., 1976. Genetics changes in natural populations of Drosophila melanogaster. Evolution, 30, 109-118.

Yamazaki T., Matsuo Y., Inoue Y., Matsuo Y., 1984. Genetic analysis of natural populations of D. melanogaster. I. Protein polymorphism. lethal gene, sterility gene, inversion polymorphism and linkage disequilibrium. Japan. J. Genetics, 59, 33-49.

Zouros E., Krimbas C.B., Tsakas S., Loukas M., 1974. Genetic versus chromosomal variation in natural populations of Drosophila subobscura. Genetics, 78, 1223-1244. 